\title{
Assessing genetic diversity of cotton cultivars using genomic and newly developed expressed sequence tag-derived microsatellite markers
}

\author{
Y. Zhang, X.F. Wang, Z.K. Li, G.Y. Zhang and Z.Y. Ma \\ North China Key Laboratory for Germplasm Resources of Education Ministry, \\ Hebei Agricultural University, Baoding, China \\ Corresponding author: Z.Y. Ma \\ E-mail: mzhy@hebau.edu.cn
}

Genet. Mol. Res. 10 (3): 1462-1470 (2011)

Received January 18, 2011

Accepted March 3, 2011

Published July 22, 2011

DOI 10.4238/vol10-3gmr1277

\begin{abstract}
Estimations of genetic diversity and of relationships between varieties are crucial for cotton breeding. The genetic diversity of 59 core cotton cultivars, most of which were collected from China's main cotton-growing areas, was analyzed based on genomic and newly developed expressed sequence tag-derived microsatellite markers, using total DNA extracted from fresh leaf tissue. Three hundred and two fragments were detected, of which 255 were polymorphic. The number of amplification products generated by each primer varied from 2 to 14, with a mean of 5.08 bands/primer. The polymorphism information content was 0.50 to 0.90 , with a mean of 0.80 . The genetic similarity coefficients were calculated and dendrograms were constructed by the unweighted pair group with arithmetic mean method; the resulting distance matrix gave a dendrogram with four main clusters. Some cultivars with similar pedigrees could be clustered. For example, Zhong206 and Shanmian4, both derived from Deltapine15, were clustered. The genetic similarity coefficient of the 59 core cultivars ranged from 0.53 to 0.99 , with a mean of 0.72 , indicating that there was a relatively high level of genetic variation.
\end{abstract}

Key words: Gossypium hirsutum; EST-SSRs; Genomic-SSRs; Genetic diversity 


\section{INTRODUCTION}

Cotton (Gossypium hirsutum L.) is one of the most important fiber crops in the world. Many successful cotton cultivars have been developed from closely related parents. Pressure for higher productivity in cotton farming has repeatedly stimulated the same gene pool and led to a narrow genetic base (Iqbal et al., 1997), which is hindering breeding programs worldwide. However, many germplasm sources still remain underused. It is necessary to dissect and exploit the natural genetic diversity conserved within cotton germplasm collections (Abdalla et al., 2001).

Assessment of the genetic diversity of cotton cultivars is essential in breeding strategies, such as the characterization of individuals, accessions, and for the choice of parental genotypes in breeding programs. For any meaningful plant-breeding program, accurate determination of genetic diversity and portioning within and between gene pools is an essential step for effectively utilizing germplasm resources. An accurate estimation of genetic diversity can be invaluable in the selection of diverse parental combinations to generate segregating progenies with maximum genetic variability and introgressing desirable traits from diverse or wild germplasm into the available cultivars to broaden the genetic base (Ulloa et al., 2007). Traditional estimation of genetic diversity was based on the morphological and biochemical markers, which may be affected by both environmental and genetic factors. Recently, various molecular marker techniques have developed into powerful tools for diversity analysis and establishing relationships between cultivars. Among these, molecular genetic techniques using DNA polymorphism have been increasingly used to characterize and identify a novel germplasm for use in the crop breeding process (Zhu et al., 2003).

For research involving cotton ( $G$. hirsutum L.), the random amplified polymorphic DNA (RAPD) technique was once the most widely used molecular method owing to its speedy process and simplicity (Multani and Lyon, 1995), and some cotton germplasm had been characterized previously with restriction fragment length polymorphisms (RFLPs) (Brubaker and Wendel, 1994). However, the levels of polymorphism detected by the above methods were generally low. These marker types were difficult to scale up for genotyping larger germplasm collections efficiently (Liu et al., 2000a). DNA marker systems for germplasm genotyping must be accurate, highly informative, amendable to automation, and cost-effective.

Simple sequence repeats (SSRs) are considered to be ideal and friendly tools for such studies as they are polymerase chain reaction (PCR)-based markers, genetically defined, typically co-dominant and uniformly dispersed throughout plant genomes (Morgante et al., 2002; Turkoglu et al., 2010). For these reasons, SSRs have become an important marker system in cultivar fingerprinting, diversity research and molecular mapping (Reddy et al., 2001). Also the loci of SSR markers are highly transferable across species (>50\%) especially within a genus (Saha et al., 2004). In addition, SSR markers derived from expressed sequence tags (ESTSSRs) are likely to be even more transferable than genomic SSRs because they are located in the transcribed regions of the genome (Park et al., 2005). Sometimes an EST-SSR marker may be a part of functional gene itself. These qualities have drawn much more attention to marker-assisted selection and comparative mapping in cotton breeding. Molecular studies of the genetic diversity in cultivated cotton had generally indicated low genetic diversity and many germplasm resources still remain underused (Tatineni et al., 1996; Iqbal et al., 1997). Thus, more study needs to be carried out. This will assist in maximizing the selection of diverse parent cultivars and broadening the germplasm base of cotton breeding programs in the 
future. The objectives of this study are 1) to evaluate the genetic diversity among selected cotton cultivars, and 2) to provide essential information for future marker-facilitated breeding and to facilitate the use of germplasms in cotton.

\section{MATERIAL AND METHODS}

\section{Plant materials}

The 59 cultivars, called core cotton collections, used in this study were selected from more than two hundred G. hirsutum cultivars provided by the Cotton Genetics and Breeding Institute, Agricultural University of Hebei. These cultivars possess many agronomically important characters such as fiber properties, resistance to diseases, pests, or low gossypol content.

\section{Genomic DNA isolation}

Seeds were germinated in 10 -cm plastic pots in a greenhouse at $28^{\circ} / 25^{\circ} \mathrm{C}$ on a 12 -h light/12-h dark cycle, with $80 \%$ relative humidity for about 1 week. Fresh leaves from at least 20 plants of each cultivar were bulked for DNA extraction. Total genomic DNA was extracted on the basis of Zhang's CTAB method (Zhang and Stewart, 2000). The purity and concentration of DNA were determined by agarose gel electrophoresis and spectrophotometric analysis. All DNA samples were diluted to a working concentration $(50 \mathrm{ng} / \mu \mathrm{L})$. Stock DNA samples were stored at $-20^{\circ} \mathrm{C}$ and working DNA samples at $4^{\circ} \mathrm{C}$ until PCR amplification.

\section{Primer selection and SSR analysis}

We made a primary survey among eight cotton cultivars on the basis of phenotypic polymorphism and pedigree information on selected markers to be used for investigating the 59 cotton cultivars. Details of the SSR markers and primer sequences can be found on the Cotton Microsatellite Database at http://www.cottonmarker.org.

PCR was performed in a total volume of $10 \mu \mathrm{L}$ containing $1.0 \mu \mathrm{L}(50 \mathrm{ng})$ genomic DNA, $1.0 \mu \mathrm{L}$ 10X PCR buffer (containing $\left.\mathrm{Mg}^{2+}\right), 0.8 \mu \mathrm{L} 2.5 \mathrm{mM}$ dNTP, $0.5 \mu \mathrm{L}$ forward primer ( $10 \mathrm{ng} /$ $\mu \mathrm{L}), 0.5 \mu \mathrm{L}$ reverse primer $(10 \mathrm{ng} / \mu \mathrm{L}), 0.2 \mu \mathrm{L}$ Taq DNA polymerase (TaKaRa, China) and $6.0 \mu \mathrm{L}$ $\mathrm{ddH}_{2} \mathrm{O}$. PCR amplification was carried out under the following conditions: one cycle consisting of $5 \mathrm{~min}$ at $95^{\circ} \mathrm{C}$ for strand separation, followed by 35 cycles of $45 \mathrm{~s}$ at $94^{\circ} \mathrm{C}$ for denaturation, 45 $\mathrm{s}$ at $57^{\circ} \mathrm{C}$ for annealing and $1 \mathrm{~min}$ at $72^{\circ} \mathrm{C}$ for primer extension. Finally, one cycle of $10 \mathrm{~min}$ at $72^{\circ} \mathrm{C}$ was used for final extension. PCR products were separated on $10 \%$ native polyacrylamide $(\mathrm{w} / \mathrm{v})$ gels at constant power $(180 \mathrm{~V})$ in $1 \mathrm{X}$ TBE running buffer, and DNA bands were visualized by the silver-staining method (Zhang et al., 2000). Finally, the gel was photographed under white/UV light attached to a gel documentation system (Bio-Rad, Hercules, CA, USA). A 50-bp DNA ladder (Fermentas, GeneRuler ${ }^{\mathrm{TM}}$, \#SM0371) was spotted on each gel as a fragment length standard. Fragments were determined visually by comparison with the DNA ladder.

The bands produced by SSR markers were scored visually: each allele was scored as present (1) or absent (0) for each of the SSR loci. Genetic diversity was calculated at each locus for allele polymorphism information content (PIC) (Anderson et al., 1993), with the CERVUS version 2.0 program based on allelic frequencies among all 59 cultivars analyzed. 
PIC values of each locus were calculated as: $\mathrm{PIC}_{\mathrm{j}}=1-\sum \mathrm{P}_{\mathrm{lj}}^{2}$, where $\mathrm{P}_{\mathrm{lj}}$ is the frequency if the $\mathrm{l}^{\text {th }}$ allele for locus $j$ is summed over its $L$ alleles. Markers were classified as informative when PIC $\geq 0.5$. The data matrix was also converted to a matrix of similarity values $(F)$ using the formula: similarity $(\mathrm{F})=2 \mathrm{~N}_{\mathrm{ab}} /\left(\mathrm{N}_{\mathrm{a}}+\mathrm{N}_{\mathrm{b}}\right)$, where $N_{a}$ and $N_{b}$ are the numbers of fragments in genotypes $a$ and $b$, respectively, and $N_{a b}$ is the number of fragments shared by genotypes $a$ and $b$ (Nei and $\mathrm{Li}, 1979)$. The results of similarity coefficients were used to evaluate the relationships among cultivars with a cluster analysis using an unweighted pairgroup method with arithmetic averages (UPGMA). The analysis was plotted in the form of a dendrogram. All computations were carried out with appropriate procedures of the package NTSys 2.1 software (Rohlf, 2000).

\section{RESULTS}

\section{SSR marker analysis}

According to the screening, 40 of 200 primers were chosen on the basis of their ability to detect the polymorphisms and production of the reliable and clear banding patterns (Figure 1A). Most of the selected SSR markers were newly developed EST-SSRs, which belonged to 15 linkage groups of cotton.

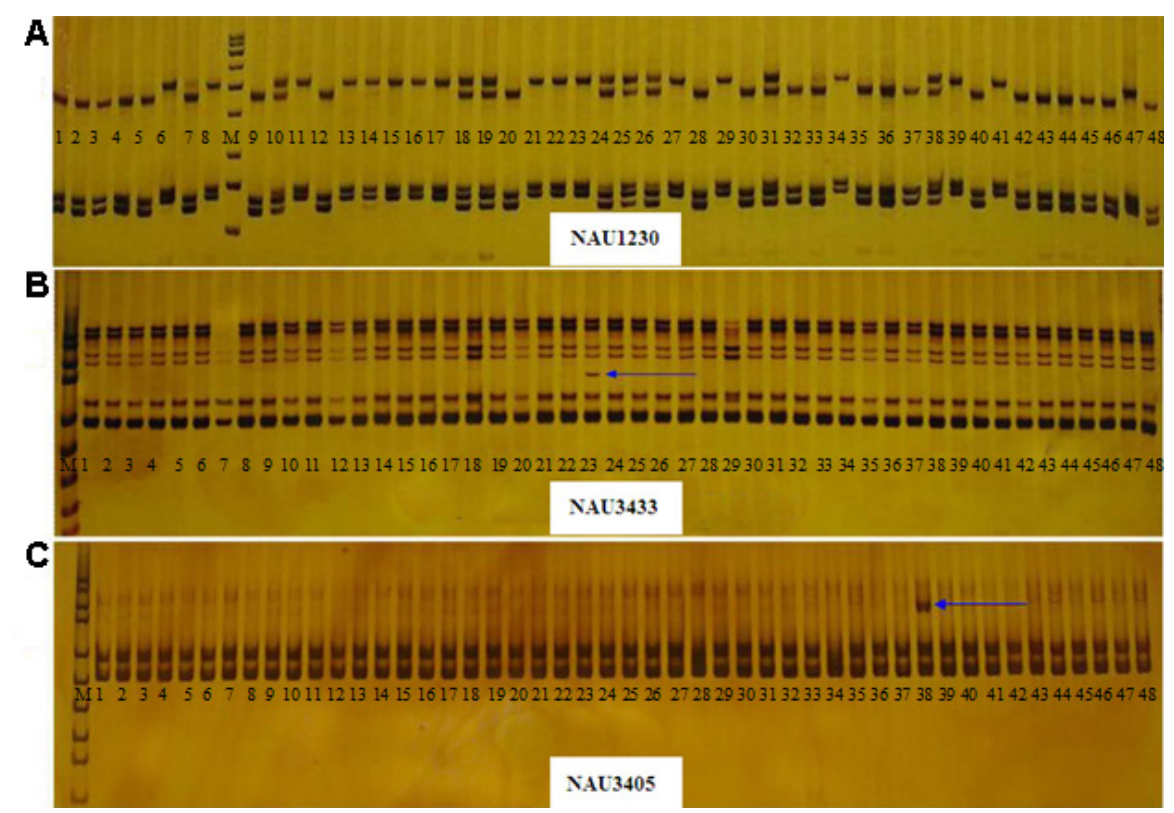

Figure 1. PCR amplification patterns for microsatellite markers (NAU1230, NAU3433, and NAU3405) in part cotton cultivars (lanes 1-48). Lane $M=$ DNA marker. The arrows represent the species-specific SSR markers generated from NAU3405 and NAU3433.

Forty selected primers produced a total of 302 fragments among the 59 cotton cultivars, of which 255 fragments (84.43\%) were polymorphic (Table 1). These SSR loci were distributed mainly on cotton chromosomes $1,4,5,6,7,8,9,11,13,15,19,21,22,23$, and 25. 
This indicates that the variations of SSR alleles were dispersed throughout the entire cotton genome. Each of the 40 primers varied greatly in their ability to resolve variability among cultivars. Several primers generated more markers, while others generated only a few. The number of amplification products generated by each primer varied from 2 to 14 with an average of 5.08 bands/primer. The primer BNL3089 gave the highest number of polymorphic bands (14), while the lowest number of polymorphic bands (2) was produced using the BNL1551 primer. The average number of polymorphic fragments per primer among 59 cotton cultivars was 4.37, higher than that found in cotton by Gutiérrez et al. (2002), who used 60 pairs of polymorphic primers to amplify 69 loci, resulting in a total of 139 alleles and an average of 2 alleles per locus, and lower than that found in cotton by Liu et al. (2000a), who used 56 polymorphic primer pairs to amplify 62 cotton loci and produced a total of 325 alleles with an average of 5 alleles per locus. Conflicting reports on the extent of observed polymorphism in cotton in different studies could be attributed to the nature of the genetic material under investigation.

Table 1. A set of 40 SSRs for the study of genetic diversity of Gossypium hirsutum cultivars.

\begin{tabular}{|c|c|c|c|c|c|c|c|c|}
\hline Primer code & Chromosome & Total band & $\begin{array}{l}\text { Polymorphic } \\
\text { fragments }\end{array}$ & $\begin{array}{l}\text { Polymorphic } \\
\text { rate }\end{array}$ & PIC & $\mathrm{H}^{\prime}$ & $\begin{array}{l}\text { Effective number } \\
\text { of alleles }\end{array}$ & $\begin{array}{c}\text { Discrimination } \\
\text { power }\end{array}$ \\
\hline BNL1053 & & 10 & 10 & 1.000 & 0.615 & 2.408 & 2.598 & 2 \\
\hline BNL1414 & A9/9 & 6 & 2 & 0.333 & 0.818 & 1.735 & 5.494 & - \\
\hline BNL1551 & D11/21 & 2 & 2 & 1.000 & 0.495 & 0.668 & 1.979 & - \\
\hline BNL1672 & A9/9 & 10 & 10 & 1.000 & 0.882 & 2.205 & 8.497 & 6 \\
\hline BNL2634 & & 7 & 4 & 0.571 & 0.829 & 1.829 & 5.860 & 3 \\
\hline BNL3031 & A9/9 & 10 & 10 & 1.000 & 0.878 & 2.191 & 8.206 & 3 \\
\hline BNL3089 & & 14 & 14 & 1.000 & 0.883 & 2.343 & 8.549 & 6 \\
\hline BNL3255 & $\mathrm{A} 4 / 4$ & 5 & 2 & 0.400 & 0.768 & 1.501 & 4.312 & - \\
\hline BNL3649 & $\mathrm{A} 8 / 8$ & 10 & 10 & 1.000 & 0.896 & 2.281 & 9.589 & 4 \\
\hline BNL4108 & D5/19 & 12 & 12 & 1.000 & 0.861 & 2.137 & 2.176 & 2 \\
\hline NAU895 & $\mathrm{A} 6 / 6$ & 8 & 7 & 0.875 & 0.851 & 1.92 & 6.691 & 1 \\
\hline NAU905 & & 7 & 6 & 0.857 & 0.488 & 1.683 & 4.712 & 5 \\
\hline NAU1037 & $\mathrm{A} 1 / 1,25$ & 6 & 4 & 0.667 & 0.805 & 1.701 & 5.128 & 1 \\
\hline NAU1042 & $\mathrm{A} 8 / 8$ & 5 & 4 & 0.800 & 0.754 & 1.486 & 4.065 & 1 \\
\hline NAU1048 & $\mathrm{A} 5 / 5$ & 6 & 5 & 0.833 & 0.810 & 1.721 & 5.261 & 1 \\
\hline NAU1093 & $\mathrm{A} 7 / 7$ & 7 & 7 & 1.000 & 0.844 & 1.899 & 6.410 & 12 \\
\hline NAU1103 & & 7 & 6 & 0.857 & 0.844 & 1.905 & 6.396 & 3 \\
\hline NAU1190 & & 9 & 9 & 1.000 & 0.865 & 2.089 & 7.417 & 9 \\
\hline NAU1230 & & 5 & 4 & 0.800 & 0.786 & 1.579 & 4.682 & - \\
\hline NAU1255 & & 5 & 4 & 0.800 & 0.761 & 1.507 & 4.815 & 1 \\
\hline NAU1369 & & 7 & 6 & 0.857 & 0.806 & 1.759 & 5.163 & 1 \\
\hline NAU2083 & & 8 & 7 & 0.875 & 0.816 & 1.867 & 5.431 & 2 \\
\hline NAU2140 & $\mathrm{A} 1 / 1,25$ & 5 & 5 & 1.000 & 0.54 & 0.883 & 2.176 & 1 \\
\hline NAU2190 & $\mathrm{A} 5 / 5$ & 8 & 8 & 1.000 & 0.821 & 1.729 & 5.598 & 4 \\
\hline NAU2238 & A $6 / 6$ & 8 & 8 & 1.000 & 0.787 & 1.764 & 4.687 & 1 \\
\hline NAU3236 & A11/11 & 5 & 2 & 0.400 & 0.775 & 1.536 & 4.438 & - \\
\hline NAU3254 & $\mathrm{A} 1 / 1,25$ & 9 & 8 & 0.889 & 0.856 & 2.036 & 6.964 & 4 \\
\hline NAU3414 & D9/23 & 5 & 5 & 1.000 & 0.793 & 1.592 & 4.826 & 2 \\
\hline NAU3433 & D7/15 & 6 & 5 & 0.833 & 0.774 & 1.550 & 4.432 & 4 \\
\hline NAU3639 & & 5 & 3 & 0.600 & 0.765 & 1.524 & 4.262 & 1 \\
\hline NAU3995 & & 12 & 12 & 1.000 & 0.903 & 2.410 & 10.341 & 10 \\
\hline NAU5013 & A $9 / 9$ & 8 & 8 & 1.000 & 0.720 & 1.434 & 3.569 & 2 \\
\hline NAU5046 & $\mathrm{D} 4 / 22$ & 5 & 5 & 1.000 & 0.782 & 1.563 & 4.597 & - \\
\hline NAU5064 & $\mathrm{A} 1 / 1$ & 6 & 4 & 0.667 & 0.798 & 1.670 & 4.941 & - \\
\hline NAU5107 & $\mathrm{A} 1 / 1,25$ & 9 & 7 & 0.778 & 0.849 & 1.989 & 6.612 & 5 \\
\hline DPL378 & $\mathrm{D} 9 / 23$ & 7 & 3 & 0.429 & 0.847 & 1.903 & 6.518 & 1 \\
\hline DPL528 & & 13 & 10 & 0.769 & 0.901 & 2.415 & 10.079 & 7 \\
\hline DPL570 & 11 & 10 & 6 & 0.600 & 0.882 & 2.198 & 8.455 & 2 \\
\hline DPL679 & A $9 / 9$ & 6 & 3 & 0.500 & 0.793 & 1.649 & 4.836 & - \\
\hline MUSS162 & 3 & 9 & 8 & 0.889 & 0.842 & 1.960 & 6.336 & 2 \\
\hline
\end{tabular}

$\mathrm{PIC}=$ polymorphism information content; $\mathrm{H}^{\prime}=-\sum \mathrm{PiLnPi}$. 
The PIC value calculated to estimate the informativeness of each primer varied from 0.50 to 0.90 with an average of 0.80 (Table 1), most of which gathered around the range of 0.76 to 0.90 (Figure 2). In the research of Liu et al. (2000b), the PIC value varied from 0.05 to 0.829 (average 0.31 ) calculated by the same method, and the value found in Lacape et al. (2007) ranged from 0.08 to 0.89 (average 0.55 ). The fact that our PIC values were higher than both of the above indicated that the cultivars used in our study might have a relatively abundant genetic base.

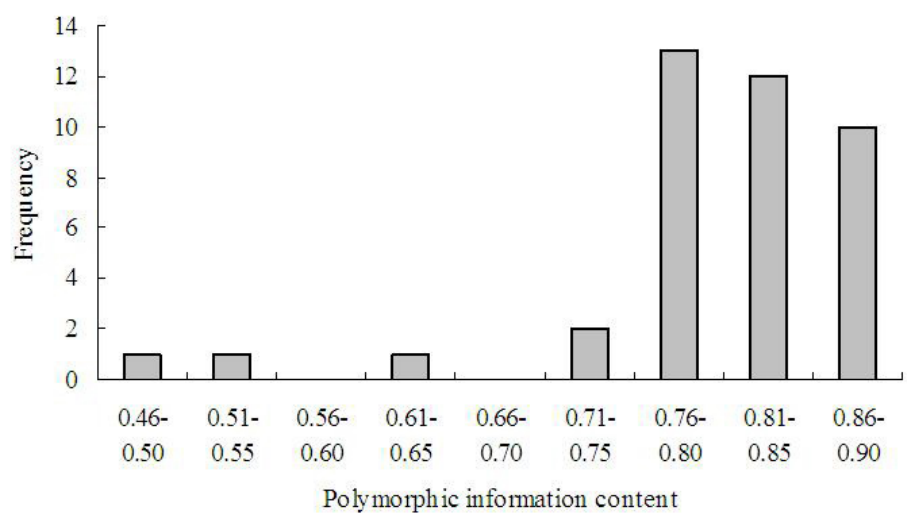

Figure 2. Distribution of polymorphic information content scores for 40 SSR markers.

We propose a set of SSR markers for priority use in tetraploid species diversity research. The loci were selected based both upon their high level of informativeness (PIC $\geq 0.5$ ) and the production of distinct bands on the gels. The set of 40 SSRs was sufficient for studies of genetic diversity of G. hirsutum. Species-specific SSR markers were generated from NAU3405 and NAU3433. Both of these markers could discriminate Jinmian14 and Zhongmiansuo12 uniquely from other cultivars, respectively (Figure 1B,C).

\section{Genetic diversity and clustering analyses}

Genetic similarity coefficients among all 59 cultivars ranged from 0.53 to 0.99 , and the maximum, 0.99, was between entry Zhongmiansuo19 and Zhongmiansuo20, which were bred by the same institution, and might mean they were closely related. With the exception of Zhongwu151 and Jimian26, pairwise genetic similarity coefficients ranged from 0.53 to 0.95 , indicating that the cultivars used in our study had a vast genetic base. Among the 59 accessions, ISABC2 was excellent because its average similarity to other accessions was 0.64 , indicating a large variability in genomic constitution. The UPGMA analysis distributed the 59 genotypes into four clusters on the basis of the Dice similarity coefficients (Figure 3). Cluster I had nine entries, which accounted for $13.8 \%$ of the total materials. Among these, Jiwu239, Mianwu4176, Zhade3, and Zhongwu151 all belong to glandless cotton. Cluster II carried 42 genotypes, of which bred by the same institution were clustered in the same group, such as Zhongmiansuo19 and Zhongmiansuo20; Zhongmiansuo 12 and Zhongmiansuo 23. There are seven members in Cluster III. Basal position of the phylogenic tree was occupied by ISABC2, 
which was made up of group V. As shown above, the ISABC2 had the highest polymorphism as revealed by microsatellite analysis in this study.

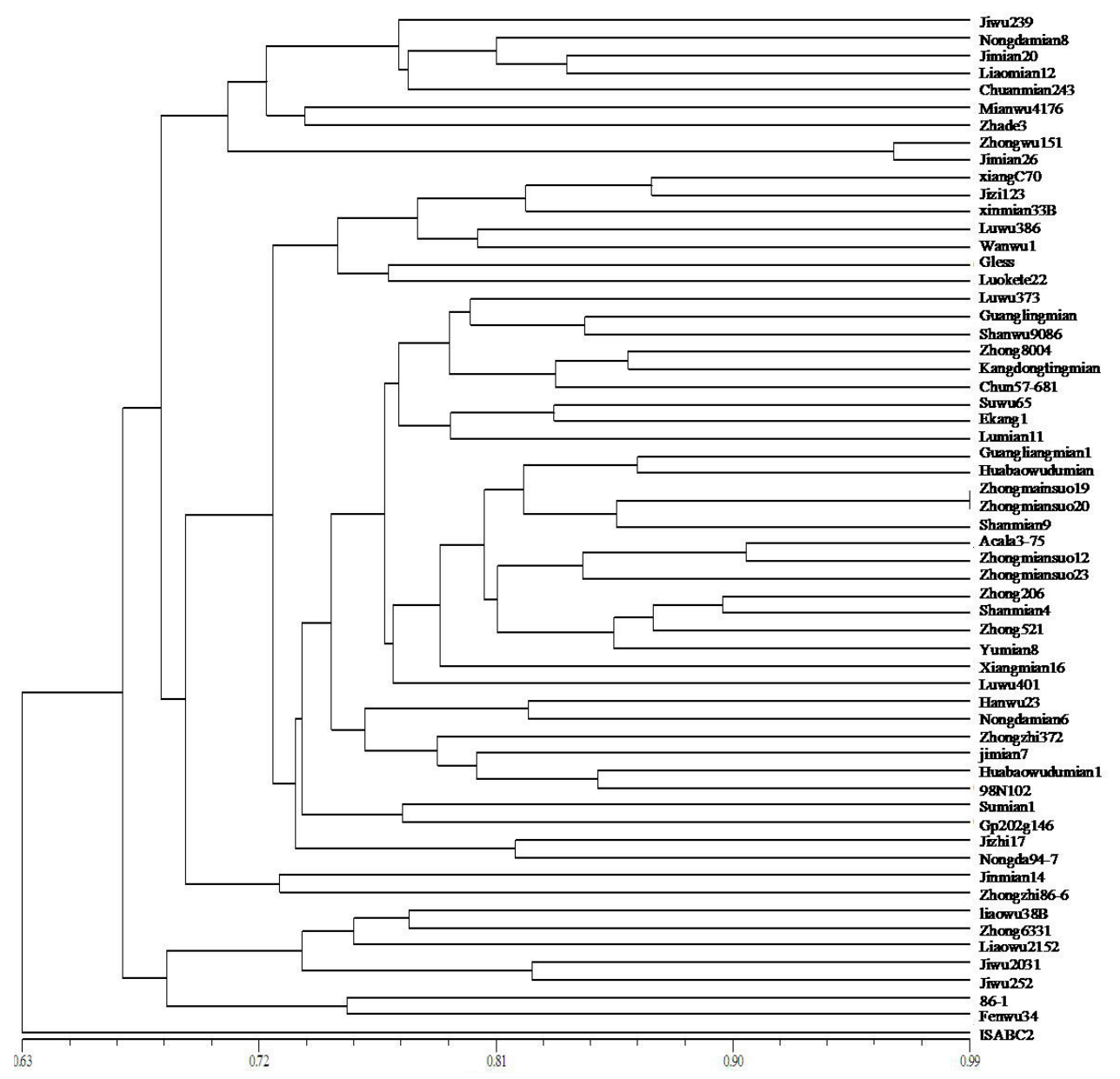

Figure 3. An UPGMA dendrogram of 59 cultivars based on SSR data.

\section{DISCUSSION}

Gossypium hirsutum is an invaluable gene pool for improving modern cotton cultivars. A systematic genetic assessment of the gene resources would help to decrease encumbrances and construct a core germplasm collection, which is significant for making use of these gene resources efficiently in cotton breeding. The use of molecular genetic markers will help to clarify the relationship among cotton germplasm. For instance, Liu et al. (2006) clustered 39 accessions of $G$. arboretum into 7 groups on the basis of GS values ranging from 0.58 to 0.95 and showed a vast genetic base in Chinese G. arboretum. Sun et al. (2009) analyzed 61 
colored-cotton lines on the basis of SSR markers, showing that the genetic background of colored cotton with elite properties was narrow. In this study, 59 cultivars were evaluated for their genetic diversity using SSR technology. Calculation of pairwise genetic similarity coefficients suggested that except for Zhongmiansuo19 and Zhongmiansuo20, which were closely related with a genetic similarity coefficient of 0.99 , and most of the $G$. hirsutum cultivars examined were genetically diversified (the coefficient ranged from 0.58 to 0.95 with an average of 0.72 ). Combining the use of these cultivars with those of lower genetic similarity can enhance our breeding efficiency in future cotton improvement programs.

Most of the selected SSR markers were newly developed EST-SSRs and probably revealed much more new polymorphic loci. These markers belonged to 19 linkage groups of cotton (Rong et al., 2004). The advantage of mapped markers is that different regions covering the entire genome can be selected and overrepresentation of certain regions can be avoided, thus leading to more accurate estimates of genetic similarities between the individuals. Besides, localization of these markers on the chromosomes would be useful for keeping track of important traits that need to be transferred. Earlier, some research had emphasized that for genetic diversity studies, markers should be chosen based on their map locations to ensure good genome coverage and reduce the marker sampling errors (Monica et al., 2004). In addition, SSR markers derived from expressed sequence tags (EST-SSRs) are likely to be more transferable than genomic SSRs because they are a part of the transcribed regions of DNA (Park et al., 2005). Transcribed regions are more conserved across species and genera, thus EST-SSRs can be used for comparative mapping (Saha et al., 2004).

In this study, the genetic similarity among genotypes was calculated as being between 0.53 and 0.99 , also emphasizing the power of SSR markers in detecting polymorphisms. And two species-specific SSR markers were found, which would be available for introgression studies where breeders want to transfer some desirable traits from one species to another.

A relatively narrow genetic base in cotton (G. hirsutum) breeding germplasm had been reported and our research showed a more or less similar result (Liu et al., 2006; Murtaza, 2006; Wang et al., 2007). The reason for the narrow genetic base may be as follows: some outstanding genes were utilized repeatedly during breeding; pressure for higher productivity in cotton farming had made the selection range narrower, and several germplasm sources still remain underused. Thus the new cultivars would be different at some loci if any. So more research is needed for evaluating and exploiting the diverse cultivars. A systematic genetic assessment of the gene resources will also help to decrease redundancy and construct a core germplasm collection, which is crucial for use of these genetic resources in cotton breeding.

\section{ACKNOWLEDGMENTS}

Research supported by the Major Program of the National Natural Science Foundation of Hebei Province (\#C2006001034).

\section{REFERENCES}

Abdalla AM, Reddy OUK, El-Zik KM and Pepper AE (2001). Genetic diversity and relationships of diploid and tetraploid cottons revealed using AFLP. Theor. Appl. Genet. 102: 222-229.

Anderson JA, Churchill GA, Autrique JE, Tanksley SD, et al. (1993). Optimizing parental selection for genetic linkage maps. Genome 36: 181-186. 
Brubaker CL and Wendel JF (1994). Reevaluating the origin of domesticated cotton (Gossypium hirsutum; Malvaceae) using nuclear restriction fragment length polymorphisms (RFLPs). Am. J. Bot. 81: 1309-1326.

Gutiérrez OA, Basu S, Saha S, Jenkins JN, et al. (2002). Genetic distance among selected cotton genotypes and its relationship with F2 performance. Crop Sci. 42: 1841-1847.

Iqbal MJ, Aziz N, Saeed NA, Zafar Y, et al. (1997). Genetic diversity evaluation of some elite cotton varieties by RAPD analysis. Theor. Appl. Genet. 94: 139-144.

Lacape JM, Dessauw D, Rajab M, Noyer JL, et al. (2007). Microsatellite diversity in tetraploid Gossypium germplasm: assembling a highly informative genotyping set of cotton SSRs. Mol. Breed. 19: 45-58.

Liu D, Guo X, Lin Z, Nie Y, et al. (2006). Genetic diversity of Asian cotton (Gossypium arboretum L) in China evaluated by microsatellite analysis. Genet. Resour. Crop Evol. 53: 1145-1152.

Liu S, Saha S, Stelly D, Burr B, et al. (2000a). Chromosomal assignment of microsatellite loci in cotton. J. Hered. 91: 326-332.

Liu S, Cantrell RG, McCarty JC and Stewart JM (2000b). Simple sequence repeat-based assessment of genetic diversity in cotton race stock accessions. Crop Sci. 40: 1459-1469.

Monica AM, Robert RK, Natalie CU, William LR, et al. (2004). Genetic diversity of public inbreds of sorghum determined by mapped AFLP and SSR markers. Crop Sci. 44: 1236-1244.

Morgante M, Hanafey M and Powell W (2002). Microsatellites are preferentially associated with nonrepetitive DNA in plant genomes. Nat. Genet. 30: 194-200.

Multani DS and Lyon BR (1995). Genetic fingerprinting of Australian cotton cultivars with RAPD markers. Genome 38: 1005-1008.

Murtaza N (2006). Cotton genetic diversity study by AFLP markers. Electron. J. Biotechnol. 9: 457-460.

Nei M and Li WH (1979). Mathematical model for studying genetic variation in terms of restriction endonucleases. Proc. Natl. Acad. Sci. U. S. A. 76: 5269-5273.

Park YH, Alabady MS, Ulloa M, Sickler B, et al. (2005). Genetic mapping of new cotton fiber loci using EST-derived microsatellites in an interspecific recombinant inbred line cotton population. Mol. Genet. Genomics 274: 428-441.

Reddy OUK, Pepper AE, Abdurakhmonov I, Saha S, et al. (2001). New dinucleotide and trinucleotide microsatellite marker resources for cotton genome research. J. Cotton Sci. 5: 103-113.

Rohlf FJ (2000). NTSYS-pc: Numerical Taxonomy and Multivariate Analysis System, Version 2.1. User Guide. Exeter Software, New York.

Rong J, Abbey C, Bowers JE, Brubaker CL, et al. (2004). A 3347-locus genetic recombination map of sequence-tagged sites reveals features of genome organization, transmission and evolution of cotton (Gossypium). Genetics 166: 389-417.

Saha S, Wu J, Jenkins JN, McCarty JC Jr, et al. (2004). Effect of chromosome substitutions from Gossypium barbadense L.3-79 into G. hirsutum L. TM-1 on agronomic and fiber traits. J. Cotton Sci. 8: 162-169.

Sun DL, Sun JL, Jia YH, Ma ZY, et al. (2009). Genetic diversiry of colored analyzed by simple sequence repeat markers. Int. J. Plant Sci. 170: 76-82.

Tatineni V, Canterell RG and Davis DD (1996). Genetic diversity in elite cotton germplasm determined by morphological characteristics and RAPDs. Crop Sci. 36: 186-192.

Turkoglu Z, Bilgener S, Ercisli S, Bakir M, et al. (2010). Simple sequence repeat-based assessment of genetic relationships among Prunus rootstocks. Genet. Mol. Res. 9: 2156-2165.

Ulloa M, Brubaker C and Chee P (2007). Cotton. In: Genome Mapping \& Molecular Breeding (Kole C, ed.). Vol. 6. Technical Crops Springer, New York.

Wang X, Ma J, Yang S, Zhang G, et al. (2007). Assessment of genetic diversity among Chinese upland cottons with Fusarium and/or Verticillium wilts resistance by AFLP and SSR markers. Front. Agric. China 1: 129-135.

Zhang J and Stewart JM (2000). Economical and rapid method for extracting cotton genomic DNA. J. Cotton Sci. 4: 193-201.

Zhang J, Wu YT, Guo WZ and Zhang TZ (2000). Fast screening of microsatellite markers in cotton with PAGE silver staining. J. Cotton Sci. 12: 267-269.

Zhu LF, Zhang XL and Nie YC (2003). Analysis of genetic diversity in upland cotton (Gossypium hirsutum L.) cultivars from China and foreign countries by RAPDs and SSRs. J. Agric. Biotechnol. 11: 450-455. 